\title{
Requirements for Next Generation Business Transformation and their Implementation in 5G Architecture
}

\author{
Aleksandar Tudzarov \\ Assistant Professor \\ Faculty of Electrical Engineering, \\ Goce Delcev University - Stip, \\ R.Macedonia
}

\author{
Saso Gelev \\ Professor \\ Faculty of Electrical Engineering, \\ Goce Delcev University - Stip, \\ R.Macedonia
}

\begin{abstract}
Main focus in this paper is placed on demystifying the $5 \mathrm{G}$ paradigm from different aspects and view's. From the beginning, from the time when the term $5 \mathrm{G}$ started to emerge in scientific circles it was unclear what exactly it represents. In the scientific community, there are many papers that are trying to present views of what they think $5 \mathrm{G}$ should be. In the past, several years it can be noticed that significant evolution in defining of the term $5 \mathrm{G}$ is made. Most importantly in the past year it can be seen that requirements for $5 \mathrm{G}$ from business perspective are converging toward unified set of needed functions, functionalities and uses cases that need to be satisfied by the next generation network called 5G. Basic idea of the paper is to present the business needs from the $5 \mathrm{G}$ network coming from the network operators/endusers and link them to what it represents viable and technically implementable architecture design given in different papers in scientific community. During this process of clarification paper also presents past and future views of $5 \mathrm{G}$ network paradigm and the needs of change.
\end{abstract}

\section{General Terms}

Business packages, 5G architecture, business transformation.

\section{Keywords}

5G, business needs, NFV, SDN, Orchestration, Cloud.

\section{INTRODUCTION}

To understand the need for network evolution and defining of new network architecture it is crucial to recognize the current business needs from the network and identify the weakness and strong sides of current networks that are wanted or not needed in the new network ecosystem.

GSM (2G) was initially designed to bring mobility for voice communications. The addition of GPSR $(2.5 \mathrm{G})$ was the first technology that offered mobile data communication in era when internet was still at its early development. UMTS (3G) was designed to be truly multiservice technology that will be able to offer different services (voice, internet data, video) thru same network but recognized the biggest boost with the addition on HSDPA and HSUPA (3.5G) in area of offering fast (first wireless broadband) mobile data services. LTE (4G) with its current evolution to LTE-A is delivering true mobile broadband in its real sense. [1]

Developments in the industry have always followed some schemes that fit well into economy of scale for the Ecosystem of Cellular Operators, network vendors \& device vendors. This has been done by following the principles that enables focus of ecosystem on one particular service for a global market and targeted for mass markets assuming the existence of what one could call the "average customer". While the evolution of $2 \mathrm{G} / 3 \mathrm{G} / 4 \mathrm{G}$ followed a "one-size-fitsall" approach, business needs from the future networks are customer centric "customer is in the center of the ecosystem". In that sense $5 \mathrm{G}$ needs a change of paradigm. $5 \mathrm{G}$ should cover the divergent needs and requirements expressed by individual use cases - while still utilizing economy of scales, efficient partnering for certain use cases, energy saving opportunities by significantly improved energy efficiency.

To fulfill the business requirements, $5 \mathrm{G}$ architecture need to include certain level of flexibility to realize required use cases. In line with that, most descriptive vision for $5 \mathrm{G}$ is the "Cloud with antennas everywhere", i.e. network functions should be performed in distributed cloud data centers, only the $\mathrm{RF}$ equipment handling the analog radio part or the fixed access lower layers will be separated out in dedicated HW. In that sense, Network Function Virtualization (NFV) and Software Defined Networking (SDN) will play a key role. SDN will play a major role as enabler for dynamic service creation and control, spanning the entire network from UE to the Data Center de-coupling of user plane from control, and the programmability of the network. [2]

\section{5G NETWORK ARHITECTURE BUISSNES REQUIREMENTS}

The need for $5 \mathrm{G}$ to support a wide variety of different use cases and business models and in the same time to be able to satisfy customer specific requirements make it impossible to design a "one-size-fits-all" 5G network, as was created for previous generations of networks serving well defined "average customer" scenario.

Instead, new flexible architecture is needed which will support an adaptation to specific combinations of use cases, business model and value propositions - called Business package. So-called Business packages require different functionalities (e.g. low/high mobility, low or normal power consumption, different security levels, different data rates and latency), that's why architecture needs the ability to dynamically create "specialized network compilation" for each of them. Figure 1, illustrates the layering of the end-toend system to support this flexibility. A Business Application Layer provides the application layer service support for the different Business packages, while the lower layer provides the $5 \mathrm{G}$ network communication system.

Building of bunch of dedicated physical infrastructures in 5G architecture for all these Business packages is not something that scales well with the costs for the technology; this would 
be even more inefficient than defining a "catch-all" architecture capturing all known requirements as far as possible. The solution shall instead be to use a cloud technology to create multiple virtual networks on a single physical infrastructure (the Infrastructure Resources Layer). The user devices are connected via multiple (fixed or mobile) access technologies, depending on use case and device capabilities.

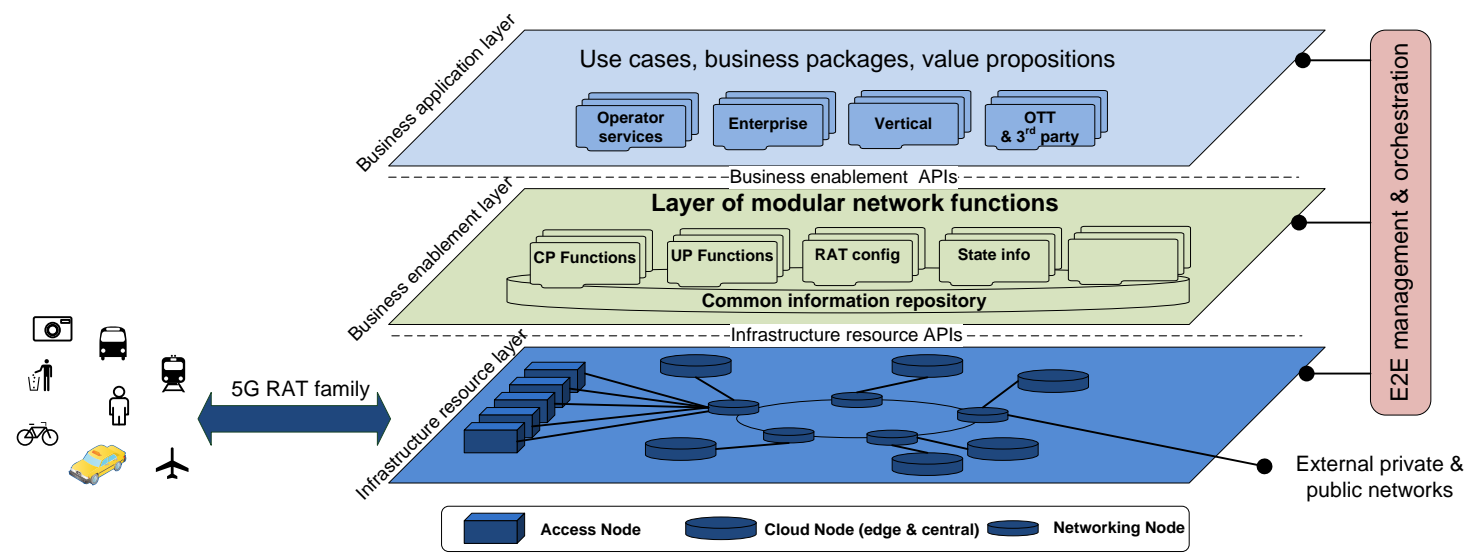

Fig.1 5G business architecture

As we cannot define today all Business packages and all required network functions for the entire lifetime of $5 \mathrm{G}$, the Business packages need to be extendible during the lifetime of 5G. E2E Management \& Orchestration offers the traditional OSS and SON functions as well as a cloud orchestration functions that automatically collect, configure, implement and then manage the various functions needed in support of business package.
The Network Infrastructure shall use as far as possible Commercial-Off-The-Shelve HW to be able to offer cost effective implementation and best relation value for the money. [3], [4]

\subsection{Use Cases requirements from 5G}

The range of use cases for $5 \mathrm{G}$ can be represented as in the six sets shown in the Table 1 below. [2]

Table 1. Use Cases and Use Case Types identified for 5 G network

\begin{tabular}{|c|c|c|}
\hline Use Case Type & Use Cases & Examples \\
\hline $\begin{array}{l}\text { Personal/Social Interaction in } \\
\text { Connected Society }\end{array}$ & $\begin{array}{c}\text { 1.Tactile Internet } \\
\text { 2.Multi user gaming } \\
\text { 3.Augmented and Virtual reality }\end{array}$ & $\begin{array}{ll}\text { - } & 50 \text { Mbps everywhere } \\
\text { - } & \text { Dense Urban Society } \\
\text { - } & \text { Pervasive video } \\
\text { - } & \text { High speed train } \\
\text { - } & \text { Smart Office } \\
\text { - } & \text { Tactile Internet } \\
\text { - } & \text { Operator Cloud Services } \\
\end{array}$ \\
\hline Massive Internet of Things & $\begin{array}{l}\text { 1.Low bit rate } \\
\text { 2. High bit rate }\end{array}$ & $\begin{array}{ll}\text { - } & \text { Smart Underwear } \\
\text { - } & \ldots\end{array}$ \\
\hline Critical \& Safety - Lifeline & 1.Emergency services & $\begin{array}{l}\text { - } \quad \text { eHealth: Extreme life critical } \\
\text { - } \quad \text { Remote object manipulation (e.g., remote } \\
\text { surgery) } \\
\text { - } \quad \text { PPDR (i.e., Natural disaster, Public Safety) } \\
\text { - } \quad \text { Controlling a disable blower for human body }\end{array}$ \\
\hline $\begin{array}{l}\text { Ultra-reliable communication } \\
(\mathrm{M} 2 \mathrm{M}, \mathrm{M} 2 \mathrm{H}, \mathrm{H} 2 \mathrm{M})\end{array}$ & $\begin{array}{c}\text { 1.Health \& Assisted Living } \\
\text { 2.Automated Industries/processes } \\
\text { 3.Remote Operation }\end{array}$ & $\begin{array}{l}\text { - } \quad \text { Automated traffic control and driving } \\
\text { - } \quad \text { Collaborative robots }\end{array}$ \\
\hline $\begin{array}{l}\text { Extreme Real-Time } \\
\text { Communication }\end{array}$ & $\begin{array}{l}\text { 1.Vehicle to } X \\
\text { 2.Extreme video }\end{array}$ & $\begin{array}{l}\text { - } \quad \text { 3D Connectivity: aircrafts } \\
\text { - } \quad \text { 3D Connectivity: post drones } \\
\text { - } \quad \text { Remote Computing }\end{array}$ \\
\hline Broadcast & $\begin{array}{l}\text { 1. local broadcast } \\
\text { 2. regional broadcast } \\
\text { 3. national broadcast }\end{array}$ & $\begin{array}{ll}\text { - } & \text { News and information } \\
\text { - } & \ldots\end{array}$ \\
\hline
\end{tabular}

Though not all use cases are equally relevant for each operator, the $5 \mathrm{G}$ system design needs to take their requirements into consideration when architecture and radio interface decisions are taken to provide the possibility of service flexibility.

\subsection{Business Models}

The most obvious role an operator can play in the future is the one of a Connectivity provider. Basically, this is a projector of existing business models into the future where a dump bitpipe is offered to retail (consumer/business) and/or 
wholesale/MVNO customers providing a best effort IP connectivity. An extension to that is the smart pipe proposition that includes the ability to offer IP connectivity which is fully configurable by customers (3rd party provider) or end users (e.g. zero rating, premium and/or guaranteed QoS).

A second role which can be played by an operator is the one of an Asset provider. Here, the operator provides the ability to offer and operate a defined part (e.g. capacity) of the network for a 3rd party provider, either national or time/location dependent. This offer includes aspects such as Infrastructure as a Service (IaaS), Network as a Service (NaaS) or Platform as a Service (PaaS). All above can be summarized as XaaS.

Another dimension of asset provisioning is real-time network sharing that refers to an operator's ability to integrate 3rd party networks in the MNO network and vice versa, based on a dynamic and context dependent policies (e.g. congestion/excess capacity policies).

A third role an operator can play in the future is the one of a Partner service provider. As an example, a partner could offer integrated multimedia communication services based on the operator's ability for 3rd parties/OTT to integrate provider communication services in their product offering. A more specialized version of that is the "tailored partner services" business model empowered by the ability for 3rd parties/OTT (e.g. content providers) to offer and create customized services specifically based on their demand and (own) content

\subsection{Value Proposition}

The close relation between users and operators is based on VALUE PROPOSITIONS. Value propositions basically are promises of an operator towards his customers but also towards partners that support the operator in providing his services. Value propositions are a key driver why a customer decides for a operator and - finally - is willing to pay. All use cases as presented earlier inherently rely on value propositions. For the time being, 9 key value attributes have been identified, namely SECURTIY, IDENTITY, PRIVACY, REAL-TIME INTERACTION, REAL-TIME EXPERIENCE, GUARANTEED RELIABILITY \& CONNECTVITY, SEAMLESS EXPERIENCE, CONTEXT VALUE CREATION, and ONE-STOP TOUCHPOINT.

\section{5G NETWORK CLOUD}

Infrastructure Resource Layer, Business Enablement Layer, and E2E Management and Orchestration entities together make up the so cold 5G Network Cloud architecture for supporting of the new Business packages, following the XaaS (everything as a service) paradigm. This new approach will be applied to all Network Functions that form part of the 5G Network Cloud, containing elements from today's Core Network, RAN and OAM domains. Vision of this architecture is presented on Figure 2.

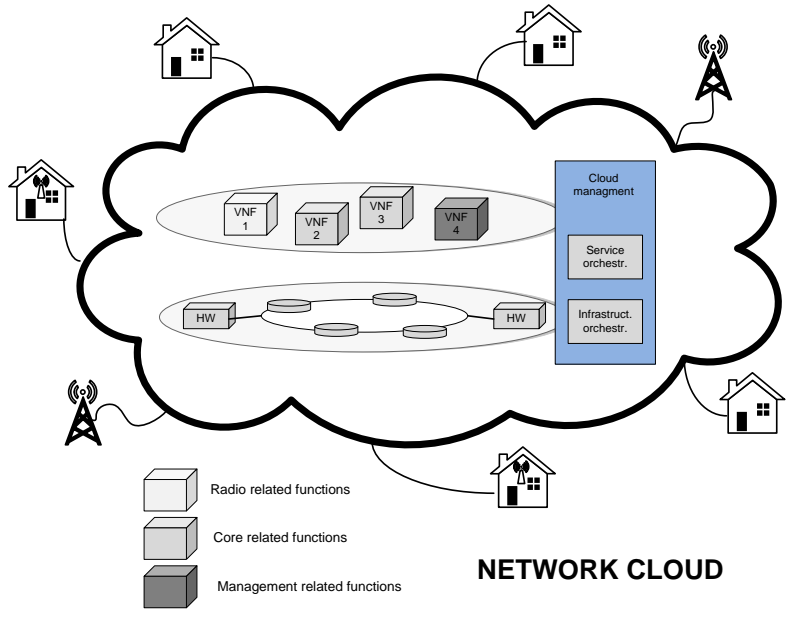

Fig. 2 Network cloud

The traditional physical separation of the domains for $\mathrm{CN}$, RAN and OAM will be superseded by the cloud flexibility to dynamically allocate functions to physical resources as befits the business scenario. Today's monolithic network boxes like the NodeB will be decomposed not only between HW and SW, but in finer grained SW functions. This should allow the application of consistent control functions within a multivendor network, and increase the re-usability of SW blocks and allow easy integration of new functionality, supporting the introduction of new radio interfaces during the system lifetime.

Non-radio network functions shall be as far as possible access interface agnostic, i.e. not require the specifics of a certain access interface. In this way, multiple radio interfaces can be supported in parallel, and new interfaces can be added during system life time. [5], [6]

The network cloud consists of two layers, the Network Infrastructure Layer (e.g. processing, storage, lines and routers / switches, data bases) and the Business Enablement Layer supporting the Virtualized Network Functions (VNFs) that run as SW blocks on this infrastructure. The Service Orchestration function provides a service chaining of VNFs to build an end-to-end service using a set of tailored function variants, as will be explained in the next section. The Infrastructure Orchestration function makes sure that VNFs are properly mapped to the respective physical infrastructure and that the end-to-end resources are allocated.

The network functions in the $5 \mathrm{G}$ architecture should be more granular than those of virtualized legacy technologies which are based upon the currently defined architecture nodes. The flexibilities required are both the flexible location of functions, and the ability to mix different function SW from different suppliers. As a minimum, the network and radio functionality should be split between control plane and user plane functions - although a further function split between loosely-coupled control functions seems desirable, e.g. between resource management and handover control. In order for network operators to be able to configure use cases, the interfacing between network functions needs to be open and vendor independent. As the composition of functions to support a use case will be SW integration rather than physical node integration, functional interfaces may not only be protocols, but could be SW integration frameworks such as Web Services or SOA, without eliminating the need for standardization. 


\section{5G ARHITECTURE TO SUPPORT BUISSNES TRANSFORMATION}

5G should contain right functional capabilities that will allow mobile operators to offer integration of all business packages. Service creation should be possible end to end by SW in a highly programmable way. $5 \mathrm{G}$ will serve the service by network slicing principle using network APIs to abstract the logical network from the underlying physical fabric. Highlevel service architecture view is shown on figure 3 .

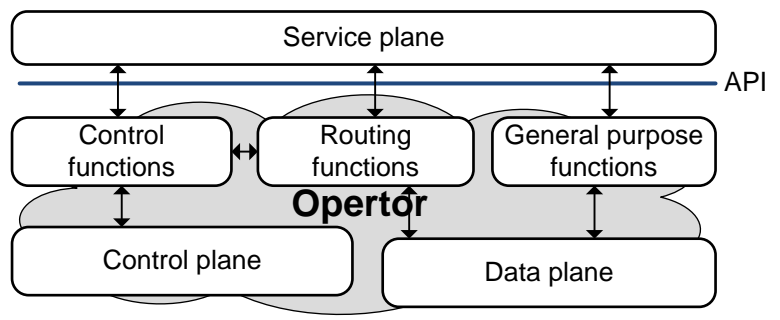

Figure 3: Service architecture view

From the business perspective, $5 \mathrm{G}$ should be able to support relationships between network operator and customer/service provider.

In the connectivity based business models, the network operator will be able to configure data flows such that only necessary network functions will be used to optimize operational and management costs. For certain machine type applications, where millions of devices with no mobility or other functional requirements and low data volumes, it would be simply too expensive to administrate the devices in the access network, and authentication modules like SIM/eSIM. This requires $5 \mathrm{G}$ to adopt network architecture with high degree of modularity such that only necessary network capabilities are used in the network slice.

On the other hand, it is essential that more tight relationships (partnerships) will be flexibly supported. 5G should provide an abstraction layer as an interface, where all different type of in-networking functionalities (control plane and data plane related) can be exposed to the application layer functions and/or service providers based on a service level agreement and by using open API. Application/Service provider will then be able to use sub-set of the network capabilities in flexible, configurable and programmable manner and use network resources depending on their service preference

5G network system should provide methods and instruments for various network sharing schemes developed to maximize the overall synergies of network sharing agreements and enable flexible business models and commercial relationships that potentially change rapidly. It means that with $5 \mathrm{G}$, it should be possible to provide enough flexibility to accommodate the capacity needs of hosted operators dynamically, on real-time basis (e.g. for capacity brokering architecture, where network resources are provided dynamically to the highest-bidding hosted operator).

To be able to create services and build up partnerships in a differentiated way, key 5G capabilities should include:

- Connectivity transparency

- $\quad$ End-to-end awareness \& QoS

- $\quad$ Security and privacy

Moreover, these capabilities along with related in-networking functionalities (e.g. mobility functions) should be exposed to higher layers through APIs. 5G may involve a collection of Radio Access Technologies (RATs) dedicated to specific services). It is required that, based on network control, user should be always connected to the RAT or combination of RATs and/or access point or combination of access points providing the best user experience without any user intervention (connectivity transparency), and in a seamless way from a user perspective.

The need for use-case specific resource/functionality allocation and service orchestration and delivery demands awareness. In a broader sense, service, data, environmental, context, proximity, and cost awareness are required with possibility to be exposed to higher architectural levels (e.g. service creation layer). QoS support needs to be explicit. Applications should not really have to know about TCP or any other protocol and it should be possible to replace transport protocols on the fly as well as allow applications to adapt if they can.

Security has been one of the fundamental services operators provide to their customers. Since $5 \mathrm{G}$ should be capable to offer services for critical sectors such as Public Safety or eHealth, utilities, the importance of guaranteeing a comprehensive and high level security is a core requirement for $5 \mathrm{G}$ systems and even stronger than previous network generations. [7], [8]

\subsection{Network Deployment, Operational and Management requirements}

In the response to challenges network operators will face in the future transformed industries and businesses, leading to significantly increased traffic, demand to support variety of use cases and wider spectrum of business/operating models, 5G must be significantly more cost efficient compared to today's $4 \mathrm{G}$ networks.

Cost efficiency relates to minimizing the Total Cost of Ownership (TCO) cost of deployment and operation of the networks infrastructure by the network operators, and the cost of terminals, for any given service offering. The TCO of a mobile network is dominated by the cost of the radio access network (RAN), therefore special attention should be given to RAN. As an example, 5G requires several system energy efficiency techniques to minimize consumption, such as turning off components, efficient pilot transmission at low load, and sleep-mode cooperation with neighbor cells, such that, combined with hardware efficiencies. Total network energy consumption is required to be reduced by $50 \%$, independent to traffic increase.

To assure maximum flexibility and scalability during the lifecycle of the technology, 5G system design should adopt functional split of network domains as well as network elements. Flexible and multi-vendor introduction of new functionalities or technologies into RAN and/or $\mathrm{CN}$ domain without HW dependencies should be possible. It should be possible to built multiple virtual networks (i.e. network slices) over the same physical infrastructure, each having different functional requirements. 5G should enable openness and multi-vendor capability at all levels.

In 5G deployments, base station density is expected to significantly increase for several reasons, including higher data volume density and the use of higher frequency spectrum. Current operations models are not prepared for emerging new technologies like 5G. The separation between Hardware- and Software will bring much more flexibility to add new functionalities in a multi-vendor and technology 
fashion. This will have a Major impact on operation. It implies reducing dramatically the complexity of Management and Operation and associated OPEX and CAPEX.

Given that $5 \mathrm{G}$ should allow connecting technology components in a plug \& play manner, also operational requirements will differ depending on technology used for different use cases. Such versatility need to be supported in efficient manner by operational and management concept.

\section{CONCLUSION}

This paper, presents the basic requirements that need to be fulfilled from next generation network as well how they fit into the new $5 \mathrm{G}$ architecture. In that manner, $5 \mathrm{G}$ needs to be designed and build for Diversity, Flexibility and efficiency. Presented architecture that is a fundament for the new so called 5G technology truly fits and is capable to deliver the needed business requirements and in that sense will support the new business transformation. Future analyses of all network requirements from all customer aspects as well as open discussion with the business needs to become regular practice until the complete process of building of $5 \mathrm{G}$ network is finished. This process should be supported by all relevant standardization bodies to produce viable and open network that will serve the needs of the customer and will satisfy business economy requirements.

\section{REFERENCES}

[1] Project METIS Deliverable D2.1 Requirements and general design principles for new air interface, 31.08.2013

[2] NGMN 5G WHITE PAPER, https://www.ngmn.org/5gwhite-paper.html, approved by NGMN board $17^{\text {th }}$ of February 2015

[3] V. O. Tikhvinskiy and G. S. Bochechka, "Conceptual aspects of $5 \mathrm{G}$ construction", Electrosvyaz, no. 10, pp. 29-33, 2013.

[4] V. G. Skrinnikov, "Future image of 5G", Electrosvyaz, no. 10 pp. 34-37, 2013.

[5] Y. Weimin, "No-Edge LTE, Now and the Future", 5G World Summit 2014 [Online]. Available http://ws.lteconference.com/.

[6] Y. Park, "5G Vision and Requirements", 5G Forum, Korea, Feb. 2014.

[7] V. O. Tikhvinskiy, G. S. Bochechka, and A. V. Minov, "LTE network monetization based on M2M services", Electrosvyaz, no. 6, pp. 12-17, 2014.

[8] "Series H: Audiovisual and multimedia systems. Infrastructure of audiovisual services - Coding of moving video. High efficiency video coding". Recommendation H.265, ITU-T 